\title{
IRCI Free Range Reconstruction for SAR Imaging With Arbitrary Length OFDM Pulse
}

\author{
Tianxian Zhang, Xiang-Gen Xia, Fellow, IEEE, and Lingjiang Kong
}

\begin{abstract}
Our previously proposed OFDM with sufficient cyclic prefix (CP) synthetic aperture radar (SAR) imaging algorithm is inter-range-cell interference (IRCI) free and achieves ideally zero range sidelobes for range reconstruction. In this OFDM SAR imaging algorithm, the minimum required $\mathrm{CP}$ length is almost equal to the number of range cells in a swath, while the number of subcarriers of an OFDM signal needs to be more than the CP length. This makes the length of a transmitted OFDM sequence at least almost twice of the number of range cells in a swath and for a wide swath imaging, the transmitted OFDM pulse length becomes long, which may cause problems in some radar applications. In this paper, we propose a CP-based OFDM SAR imaging with arbitrary pulse length, which has IRCI free range reconstruction and its pulse length is independent of a swath width. We then present a novel design method for our proposed arbitrary length OFDM pulses. Simulation results are presented to illustrate the performances of the OFDM pulse design and the arbitrary pulse length CP-based OFDM SAR imaging.
\end{abstract}

Index Terms-Cyclic prefix (CP), inter-range-cell interference (IRCI), orthogonal frequency-division multiplexing (OFDM) pulse, range reconstruction, synthetic aperture radar (SAR) imaging, waveform design.

\section{INTRODUCTION}

$\mathbf{O}$ RTHOGONAL frequency-division multiplexing (OFDM) signals are firstly presented for radar signal processing in [1], and recently studied and used in radar applications, such as moving target detection [2]-[4], low-grazing angle target tracking [5] and ultrawideband (UWB) radar

Manuscript received December 06, 2013; revised March 28, 2014 and July 06,2014 ; accepted July 11, 2014. Date of publication July 16, 2014; date of current version August 14,2014. The associate editor coordinating the review of this manuscript and approving it for publication was Prof. Yimin D. Zhang. The work of T. Zhang was supported in part by the Fundamental Research Funds for the Central Universities under Grant ZYGX2012YB008 and in part by the China Scholarship Council (CSC) and was done when he was visiting the University of Delaware, Newark. The work of X.-G. Xia was supported in part by the Air Force Office of Scientific Research (AFOSR) under Grant FA9550-12-1-0055. The work of L. Kong was supported in part by the Fundamental Research Funds of Central Universities (ZYGX2012Z001) and in part by the Program for New Century Excellent Talents in University (A1098524023901001063).

T. Zhang is with the School of Electronic Engineering, University of Electronic Science and Technology of China, Chengdu, Sichuan, China, 611731, and also with the Department of Electrical and Computer Engineering, University of Delaware, Newark, DE 19716 USA (e-mail: tianxian.zhang@gmail.com).

X.-G. Xia is with the Department of Electrical and Computer Engineering, University of Delaware, Newark, DE 19716 USA (e-mail: xxia@ee.udel.edu).

L. Kong is with the School of Electronic Engineering, University of Electronic Science and Technology of China, Chengdu, Sichuan, P.R. China, 611731 (e-mail: lingjiang.kong@gmail.com).

Color versions of one or more of the figures in this paper are available online at http://ieeexplore.iee.org.

Digital Object Identifier 10.1109/TSP.2014.2339796 applications [6]. The common OFDM signals for digital communications, such as the digital audio broadcast (DAB), digital video broadcast (DVB), Wireless Fidelity (WiFi) or worldwide inoperability for microwave access (WiMAX) signals, are also investigated for radar applications in [7]-[12]. Using OFDM signals for synthetic aperture radar (SAR) applications is proposed in [13]-[20]. In [13]-[15], an adaptive OFDM signal design is studied for range ambiguity suppression in SAR imaging. The reconstruction of cross-range profiles is studied in [18], [19]. However, all the existing OFDM SAR signal processing algorithms have not considered the feature of OFDM signals with sufficient cyclic prefix (CP) as used in communications systems. In [21], we have proposed a sufficient CP-based OFDM SAR imaging algorithm. By using a sufficient $\mathrm{CP}$, the inter-range-cell interference (IRCI) free and ideally zero range sidelobes for range reconstruction can be obtained, which provides an opportunity for high range resolution SAR imaging. On the other hand, according to our analysis, the CP length, the transmitted OFDM pulse length and the minimum radar range need to be increased with the increase of a swath width, since the sufficient $\mathrm{CP}$ length is almost equal to the number of range cells in a swath, while the number of subcarriers of the OFDM signal needs to be more than the $\mathrm{CP}$ length. Then, the transmitted OFDM sequence with sufficient $\mathrm{CP}$ should be at least almost twice of the number of range cells in a swath. Meanwhile, the $\mathrm{CP}$ sequence needs to be removed at the receiver to achieve the IRCI free range reconstruction. Thus, this algorithm may need a long transmitted pulse and suffer high transmitted energy redundancy in case of wide swath imaging, which may cause problems in some radar applications.

Although OFDM signals have been widely used in practical digital communications and studied for radar applications, the potential high peak-to-average power ratio (PAPR) of OFDM signals may cause problems for communications applications [22] and radar applications [3], because the envelope of OFDM signals is time-varying. In power amplifier of the transmitter, a constant envelope waveform can be magnified efficiently in the saturation region. However, the amplifier should be operated in the limited linear region for a time-varying signal to avoid causing nonlinear distortion. Many PAPR reduction techniques have been studied as, for example, in [23].

In this paper, we propose a sufficient CP-based OFDM SAR imaging with arbitrary pulse length that is independent of a swath width. Firstly, we establish the arbitrary pulse length OFDM SAR imaging system model by considering the feature of OFDM signals with sufficient $\mathrm{CP}$, where the CP part is all zero. We then derive a sufficient $\mathrm{CP}$-based range reconstruction 
algorithm with an OFDM pulse, whose length is independent of a swath width. To investigate the signal-to-noise ratio (SNR) degradation caused by the range reconstruction, we also analyze the change of noise power in every step of the range reconstruction. By considering the PAPR of the transmitted OFDM pulses and the SNR degradation within the range reconstruction, we propose a new OFDM pulse design method. We then present some simulations to demonstrate the performance of the proposed OFDM pulse design method. By comparing with the range Doppler algorithm (RDA) SAR imaging method using linear frequency modulated (LFM) signals, we present some simulations to illustrate the performance of the proposed arbitrary pulse length OFDM SAR imaging algorithm. We find that, with a designed arbitrary length OFDM pulse from our proposed method, this algorithm can still maintain the advantage of IRCI free range reconstruction with insignificant SNR degradation and completely avoid the energy redundancy.

The remainder of this paper is organized as follows. In Section II, we briefly recall the CP-based OFDM SAR algorithm proposed in [21] and describe the problem of interest. In Section III, we propose CP-based arbitrary pulse length OFDM SAR. In Section IV, we propose a new arbitrary length OFDM sequence design algorithm. In Section V, we show some simulation results. Finally, in Section VI, we conclude this paper.

\section{CP-BASED OFDM SAR AND PROBLEM FORMULATION}

In this section, we first briefly recall the CP-based OFDM SAR model proposed in [21] and then see its required pulse length problem. Consider the monostatic broadside stripmap SAR geometry as shown in Fig. 1. The radar platform is moving parallelly to the $y$-axis with an instantaneous coordinate $\left(0, y_{p}(\eta), H_{p}\right), H_{p}$ is the altitude of the radar platform, $\eta$ is the relative azimuth time referenced to the time of zero Doppler, $T_{a}$ is the synthetic aperture time defined by the azimuth time extent the target stays in the antenna beam. For convenience, let us choose the azimuth time origin $\eta=0$ to be the zero Doppler sample. Consider an OFDM signal with $N$ subcarriers, a bandwidth of $B \mathrm{~Hz}$, and let $\boldsymbol{S}=\left[S_{0}, S_{1}, \ldots, S_{N-1}\right]^{T}$ represent the complex weights transmitted over the subcarriers, $(\cdot)^{T}$ denotes the transpose, and $\|S\|^{2}=\sum_{i=0}^{N-1}\left|S_{i}\right|^{2}=1$. Note that, although this sequence $S_{i}$ is rather general, in [21], a pseudo random sequence $S_{i}$ with constant module is proposed to be used for achieving the optimal SNR at the receiver. Then, a discrete time OFDM signal is the inverse fast Fourier transform (IFFT) of the vector $\boldsymbol{S}$ and the corresponding time domain OFDM signal is

$$
s(t)=\frac{1}{\sqrt{N}} \sum_{k=0}^{N-1} S_{k} \exp \{j 2 \pi k \Delta f t\}, t \in\left[0, T+T_{G I}\right],
$$

where $\Delta f=\frac{B}{N}=\frac{1}{T}$ is the subcarrier spacing. $\left[0, T_{G I}\right)$ is the time duration of the guard interval that corresponds to the $\mathrm{CP}$ in the discrete time domain as we shall see later in more details and its length $T_{G I}$ will be specified later too, $T$ is the length of the OFDM signal excluding CP. Due to the periodicity of the exponential function $\exp (\cdot)$ in $(1)$, the tail part of $s(t)$ for

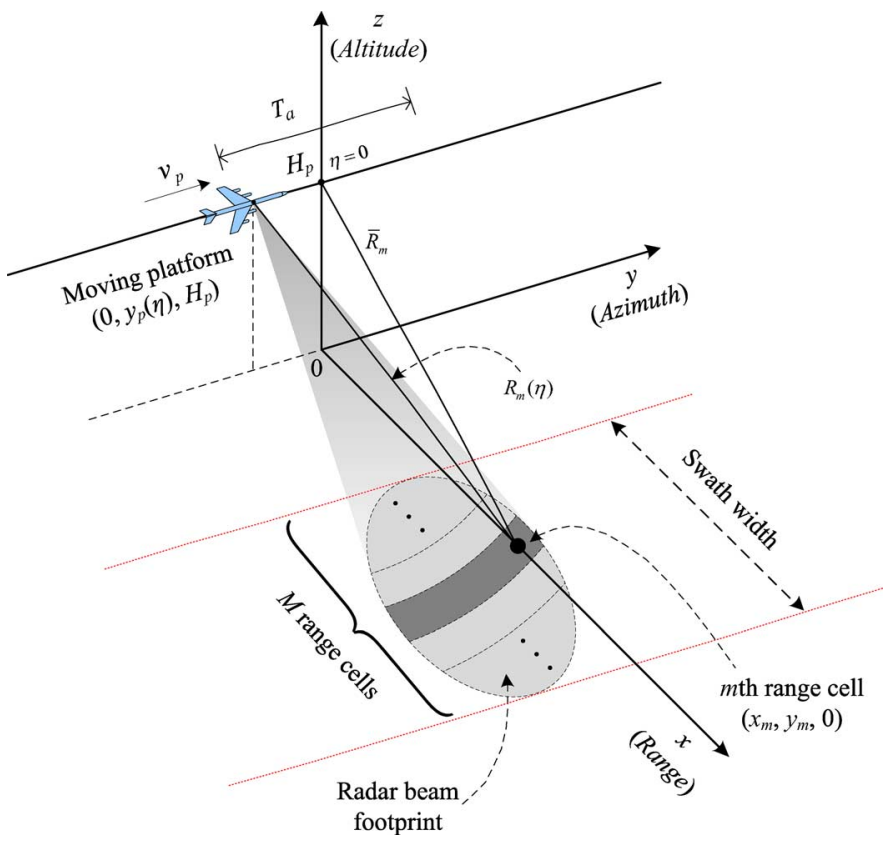

Fig. 1. Monostatic stripmap SAR geometry.

$t$ in $\left(T, T+T_{G I}\right]$ is the same as the head part of $s(t)$ for $t$ in $\left[0, T_{G I}\right)$.

After the demodulation to baseband, the complex envelope of the received signal from all the range cells in the swath can be written in terms of fast time $t$ and slow time $\eta$

$$
\begin{aligned}
u(t, \eta) & =\frac{1}{\sqrt{N}} \sum_{m} g_{m} \exp \left\{-j 4 \pi f_{c} \frac{R_{m}(\eta)}{c}\right\} \\
& \times \sum_{k=0}^{N-1} S_{k} \exp \left\{\frac{j 2 \pi k}{T}\left[t-\frac{2 R_{m}(\eta)}{c}\right]\right\}+w(t, \eta),
\end{aligned}
$$

where $f_{c}$ is the carrier frequency, $g_{m}$ is the radar cross section (RCS) coefficient caused from the scatterers in the $m$ th range cell within the radar beam footprint, and $c$ is the speed of light. $w(t, \eta)$ represents the noise. $R_{m}(\eta)=\sqrt{x_{m}^{2}+H_{p}^{2}+v_{p}^{2} \eta^{2}}$ is the instantaneous slant range between the radar and the $m$ th range cell with the coordinate $\left(x_{m}, y_{m}, 0\right)$, and $v_{p}$ is the effective velocity of the radar platform.

At the receiver, the received signal is sampled by the A/D converter with sampling interval length $T_{s}=\frac{1}{B}$ and the range resolution is $\rho_{r}=\frac{c}{2 B}=\frac{c}{2} T_{s}$. Assume that the swath width for the radar is $R_{w}$. Then, a range profile can be divided into $M=\frac{R_{w}}{\rho_{r}}$ range cells that is determined by the radar system. According to the analysis in [21], $M$ range cells correspond to $M$ paths in communications, which include one main path (i.e., the nearest range cell) and $M-1$ multipaths. In order to avoid the IRCI (corresponding to the intersymbol interference (ISI) in communications) between different range cells, the CP length should be at least equal to the number of multipaths $(M-$ 1). For convenience, we set $\mathrm{CP}$ length as $M-1$ in [21], and then the guard interval length in (1) is $T_{G I}=(M-1) T_{s}$. Notice that $T=N T_{s}$. Thus, the time duration of an OFDM pulse is $T_{o}=T+T_{G I}=(N+M-1) T_{s}$. Meanwhile, to completely avoid the IRCI between different range cells, the 
number, $N$, of the OFDM signal subcarriers should satisfy $N \geq$ $M$ as we have analyzed in [21] and also well understood in communications applications [23]. Therefore, the transmitted pulse duration $T_{o}$ is increased with the increase of the swath width. For example, if we want to increase the swath width to $10 \mathrm{~km}$, the transmitted pulse duration $T_{o}$ should be increased to about $133.3 \mu \mathrm{s}$. The pulse length here is much longer than the traditional radar pulse, which might be a problem, especially, for covert/military radar applications. Therefore, it is important to achieve OFDM SAR imaging with arbitrary pulse length that is independent of a swath width, and in the meantime it also has ideally zero IRCI. This is the goal of the remainder of this paper.

\section{CP-Based Arbitrary Pulse Length OFDM SAR}

The main idea of the following study is to generate a pulse $s(t), t \in\left[0, T+T_{G I}\right]$, such that $s(t)=0$ for $t \in\left[0, T_{G I}\right)$ and also for $t \in\left(T, T+T_{G I}\right]$ with an arbitrary $T$ for $T>T_{G I}$, and $s(t)$ is an OFDM signal in (1) for $t \in\left[T_{G I}, T\right]$. However, if the non-zero segment $s(t)$ for $t \in\left[T_{G I}, T\right]$ is directly a segment of an arbitrary OFDM signal in (1), the whole sampled discrete time sequence of $s(t), 0 \leq t \leq T+T_{G I}: s_{n}=s_{n}\left(n T_{s}\right), 0 \leq$ $n \leq N+M-2$, that is zero at the head and tail ends from the above design idea of $s(t)$, may not be from a sampling of any OFDM pulse in (1) for $t \in\left[0, T+T_{G I}\right]$. Thus, such a pulse may not be used in the IRCI free range reconstruction as in [21]. The key of this paper is to generate such a pulse $s(t)$ with the above property of zero-valued head and tail, and in the meantime, its sampled discrete time sequence $s_{n}$ is also a sampled discrete time sequence of an OFDM pulse in (1) for $t \in\left[0, T+T_{G I}\right]$. Since the non-zero pulse length is $T-T_{G I}$ and $T$ is arbitrary, the non-zero pulse length is also arbitrary and independent of a swath width. The details is given in the following subsections.

\section{A. Received Signal Model}

In order to better understand the IRCI free range reconstruction, let us first see the receive signal model. Going back to (2), for the $m$ th range cell, $R_{m}(\eta)=R_{0}(\eta)+m \rho_{r}$, where $R_{0}(\eta)$ is the instantaneous slant range between the radar and the first range cell in the swath as in [21]. For $t-\frac{2 R_{m}(\eta)}{c}=$ $t-\frac{2 R_{0}(\eta)}{c}-m T_{s}$, let the sampling be aligned with the start of the received signal after $\frac{2 R_{0}(\eta)}{c}$ seconds for the first arriving version of the transmitted signal, $u(t, \eta)$ in (2) can be converted to the discrete time linear convolution of the transmitted sequence with the weighting RCS coefficients $d_{m}$, i.e.,

$$
\tilde{u}_{n}=\sum_{m=0}^{M-1} d_{m} s_{n-m}+\tilde{w}_{n}, \quad n=0,1, \ldots, N+2 M-3,
$$

where

$$
d_{m}=g_{m} \exp \left\{-j 4 \pi f_{c} \frac{R_{m}(\eta)}{c}\right\}
$$

in which $4 \pi f_{c} \frac{R_{m}(\eta)}{c}$ in the exponential is the azimuth phase, and $s_{n}$ is the sampled discrete time sequence, $s_{n}=s\left(n T_{s}\right)$, of the transmitted pulse $s(t)$ during $t \in\left[0, T+T_{G I}\right]$ for $T=N T_{s}$ and $T_{G I}=(M-1) T_{s}$. Since the range reconstruction in the SAR imaging algorithm proposed in [21] in the following is only based on the discrete time signal model in (3), what matters in the range reconstruction is the discrete time sequence $s_{n}=s\left(n T_{s}\right)$, where $s_{n}=0$ for $n<0$. If the sequence $\boldsymbol{s}^{\prime}=\left[s_{0}, s_{1}, \ldots, s_{N+M-2}\right]^{T}$ in (3) has the following zero head and tail property:

$$
\left[s_{0}, \ldots, s_{M-2}\right]^{T}=\left[s_{N}, \ldots, s_{N+M-2}\right]^{T}=\mathbf{0}_{(M-1) \times 1},
$$

then, in terms of the range reconstruction later, the transmitted pulse $s(t)$ is equivalent to that with $s(t)=0$ for $t \in\left[0, T_{G I}\right)$ and $t \in\left(T, T+T_{G I}\right]$. It is also equivalent to an OFDM pulse in (1) such that its sampled version

$$
\begin{aligned}
s_{n}=s\left(n T_{s}\right)=\frac{1}{\sqrt{N}} \sum_{k=0}^{N-1} S_{k} \exp \left\{\frac{j 2 \pi k n}{N}\right\}, \\
n=0,1, \ldots, N+M-2,
\end{aligned}
$$

has the property (5).

In summary, our proposed transmitted pulse of an arbitrary length $s(t)$ of non-zero is that $s(t)=0$ for $t \in\left[0, T_{G I}\right)$ and $t \in$ $\left(T, T+T_{G I}\right]$ and $s(t)$ has the OFDM form (1) for $t \in\left[T_{G I}, T\right]$ with an arbitrary $T$ of $T>T_{G I}$, where the sampled version $s_{n}$ of the analog waveform/pulse in (1) satisfies the zero head and tail property (5). Note that, since $T-T_{G I}$ is arbitrary and $s(t)$ is only non-zero in the interval $\left[T_{G I}, T\right]$, its non-zero pulse length is arbitrary. Furthermore, since for the sequence $\boldsymbol{s}^{\prime}$, its both head and tail parts are the same of all zeroes with length $M-1$, the head part is a CP of the tail part and thus it fits to the sufficient CP-based SAR imaging proposed in [21].

Based on the above analysis, in what follows, we assume that an OFDM pulse in (1) satisfies the zero head and tail property (5) for its sampled discrete time sequence $s_{n}$ and thus, it is equivalent to a pulse of length $T-T_{G I}$ as described above in terms of the range reconstruction. So, for convenience, we may use these two kinds of pulses interchangeably. Note that the reason why these two kinds of analog waveforms are not the same is because a non-zero OFDM signal in (1) can not be all zero for $t$ in any interval of a non-zero length.

From (6), it is clear that the time domain OFDM sequence $s=$ $\left[s_{0}, s_{1}, \ldots, s_{N-1}\right]^{T}$ is just the $N$-point IFFT of the vector $\boldsymbol{S}=$ $\left[S_{0}, S_{1}, \ldots, S_{N-1}\right]^{T}$. In the SAR imaging algorithm proposed in [21], $N \geq M$ is required, which is the same as $T>T_{G I}$. However, from the above study, there are only $N-M+1$ non-zero values in the sequence $s$ and $N$ can be arbitrary as long as $N \geq M$. In this case, the transmitted sequence is just $\boldsymbol{s}_{t}=\left[s_{M-1}, s_{M}, \ldots, s_{N-1}\right]^{T} \in \mathbb{C}^{(N-M+1) \times 1}$. Then, the first and the last $M-1$ samples of the received signal $\tilde{u}_{n}$ in (3) do not contain any useful signal ${ }^{1}, d_{m}$. Thus, we can start the sampling at $\tilde{u}_{M-1}$ as

$$
u_{n}=\sum_{m=0}^{M-1} d_{m} s_{n-m+M-1}+w_{n}, \quad n=0,1, \ldots, N-1 .
$$

${ }^{1}$ In [21], the first and the last $M-1$ samples of the received signal $\tilde{u}_{n}$ in (3) contain received target energy (or useful signal), but they are redundant and removed at the receiver to obtain $u_{n}$ and IRCI free range reconstruction. 
Now the question is how to design such an arbitrary length pulse, which is studied next after the range reconstruction algorithm is introduced.

\section{B. Range Compression}

In this subsection, we develop the range compression according to the above OFDM received signal model. The received signal $\boldsymbol{u}=\left[u_{0}, u_{1}, \ldots, u_{N-1}\right]^{T}$ in (7) is equivalent to the following representation

$$
\boldsymbol{u}=\boldsymbol{H} \boldsymbol{s}_{t}+\boldsymbol{w},
$$

where $\boldsymbol{w}=\left[w_{0}, w_{1}, \ldots, w_{N-1}\right]^{T}$ is the noise vector and $\boldsymbol{H}$ is the $N$ by $N-M+1$ matrix:

$$
\boldsymbol{H}=\left[\begin{array}{cccc}
d_{0} & 0 & \cdots & 0 \\
d_{1} & d_{0} & \ddots & \vdots \\
\vdots & \vdots & \ddots & 0 \\
d_{M-1} & d_{M-2} & \cdots & \vdots \\
0 & \ddots & \ddots & \vdots \\
\vdots & \ddots & d_{M-1} & d_{M-2} \\
0 & \cdots & 0 & d_{M-1}
\end{array}\right]
$$

The OFDM demodulator then performs the $N$-point fast Fourier transform (FFT) on the vector $\boldsymbol{u}$ :

$$
\begin{aligned}
U_{i} & =\frac{1}{\sqrt{N}} \sum_{n=0}^{N-1} u_{n} \exp \left\{\frac{-j 2 \pi i n}{N}\right\} \\
& =D_{i} S_{i}^{\prime}+W_{i}, \quad i=0,1, \ldots, N-1,
\end{aligned}
$$

where $\left[S_{0}^{\prime}, S_{1}^{\prime}, \ldots, S_{N-1}^{\prime}\right]^{T}$ is the $N$-point FFT of the sequence $\left[\boldsymbol{s}_{t}^{T}, \mathbf{0}_{1 \times(M-1)}\right]^{T}$, a cyclic shift of the time domain OFDM sequence $s$ of amount $M-1$, i.e.,

$$
S_{i}^{\prime}=S_{i} \exp \left\{\frac{j 2 \pi i(M-1)}{N}\right\},
$$

$\boldsymbol{W}=\left[W_{0}, \ldots, W_{N-1}\right]^{T}$ is the $N$-point FFT of the noise vector $\boldsymbol{w}$, and

$$
D_{i}=\sum_{m=0}^{M-1} d_{m} \exp \left\{\frac{-j 2 \pi m i}{N}\right\}
$$

Then, the estimate of $D_{i}$ is

$$
\hat{D}_{i}=\frac{U_{i}}{S_{i}^{\prime}}=D_{i}+\frac{W_{i}}{S_{i}^{\prime}}, \quad i=0,1, \ldots, N-1 .
$$

The vector $\boldsymbol{D}=\left[D_{0}, D_{1}, \ldots, D_{N-1}\right]^{T}$ is just the $N$-point FFT of the vector $\sqrt{N}\left[d_{0}, d_{1}, \ldots, d_{M-1}, \mathbf{0}_{1 \times(N-M)}\right]^{T}$. So, the estimate of $d_{m}$ can be achieved by the $N$-point IFFT of the vector $\hat{\boldsymbol{D}}=\left[\hat{D}_{0}, \hat{D}_{1}, \ldots, \hat{D}_{N-1}\right]^{T}:$

$$
\hat{d}_{m}=\frac{1}{\sqrt{N}} \sum_{i=0}^{N-1} \hat{D}_{i} \exp \left\{\frac{j 2 \pi m i}{N}\right\}, \quad m=0, \ldots, M-1 .
$$

Then, we obtain the following estimates of the $M$ weighting RCS coefficients:

$$
\hat{d}_{m}=\sqrt{N} d_{m}+\hat{w}_{m}^{\prime}, \quad m=0, \ldots, M-1,
$$

where $\hat{w}_{m}^{\prime}$ is from the noise. In (15), $d_{m}$ can be recovered without any IRCI from other range cells.

After the range compression, combining the (2)-(4) and (15), we obtain

$$
\begin{aligned}
u_{r a}(t, \eta)=\sqrt{N} & \sum_{m=0}^{M-1} \hat{g}_{m} \delta\left(t-\frac{2 R_{m}(\eta)}{c}\right) \\
& \times \exp \left\{-j 4 \pi f_{c} \frac{R_{m}(\eta)}{c}\right\}+w_{r a}(t, \eta),
\end{aligned}
$$

where $\delta\left(t-\frac{2 R_{m}(\eta)}{c}\right)$ is the delta function with non-zero value at $t=\frac{2 R_{m}(\eta)}{c}$, which indicates that, for every $m$, the estimate $\hat{g}_{m}$ of the RCS coefficient value $g_{m}$ is not affected by any IRCI from other range cells after the range compression. The azimuth phase in the exponential is unaffected by the range compression. In summary, the above range compression provides an IRCI free range reconstruction.

Notice that unlike the processing in [21] where the first and the last $M-1$ samples of the received signal are removed and thus cause significant transmitted energy waste for a wide swath imaging, in the above range reconstruction algorithm, all the transmitted energy is used for the range compression without any waste. Since the transmitted OFDM pulse time duration is $T-T_{G I}$, the minimum radar range is $\frac{c\left(T-T_{G I}\right)}{2}$ that is also independent of a swath width. Different from [21] where the $\mathrm{CP}$ part is not zero, the pulse repetition interval $T_{\mathrm{PRI}}$ becomes

$$
T_{\mathrm{PRI}}=\frac{1}{\mathrm{PRF}}>\left(\frac{2 R_{w}}{c}+\left(T-T_{G I}\right)\right),
$$

where $R_{w}$ is the swath width and PRF is the pulse repetition frequency (PRF). We want to emphasize here that the minimum radar range and the maximum PRF of our proposed OFDM SAR in this paper are the same as those in the existing SAR systems, such as LFM SAR, when the same transmitted pulse time duration is used [24], [25].

\section{Signal-to-Interference and Noise Ratio Analysis After Range Compression}

In the above range compression, the processes of FFT in (10), estimation in (13) and IFFT in (14) are applied. Thus, it is necessary to analyze the changes of the noise power in each step of the range compression. Assume that $w_{n}$ in (7) is a complex white Gaussian variable with zero-mean and variance $\sigma^{2}$, i.e., $w_{n} \sim \mathcal{C N}\left(0, \sigma^{2}\right)$ for all $n$. Since the FFT operation is unitary, the additive noise power does not change after the process of (10). Thus, $W_{i}$ also obeys $W_{i} \sim \mathcal{C N}\left(0, \sigma^{2}\right)$ for all $i$. However, let $\bar{W}_{i}=\frac{W_{i}}{S_{i}^{\prime}}$ in (13), then the variance of $\bar{W}_{i}$ is changed to $\frac{\sigma^{2}}{\left|S_{i}\right|^{2}}$, where, from (11), $\left|S_{i}^{\prime}\right|=\left|S_{i}\right|$, and thus $\bar{W}_{i} \sim \mathcal{C N}\left(0, \frac{\sigma^{2}}{\left|S_{i}\right|^{2}}\right), i=0, \ldots, N-1$. Moreover, after the IFFT operation in (14) we have finished the range compression and the noise power of $\hat{w}_{m}^{\prime}$ in (15) is 
$\frac{\sigma^{2}}{N} \sum_{i=0}^{N-1}\left|S_{i}\right|^{-2}$ and in the meantime $\hat{w}_{m}^{\prime}$, that follows the distribution $\mathcal{C N}\left(0, \frac{\sigma^{2}}{N} \sum_{i=0}^{N-1}\left|S_{i}\right|^{-2}\right)$, is equivalent to the noise $w_{r a}(t, \eta)$ in (16). Thus, from (15), we can obtain the SNR of the $m$ th range cell after the range compression as,

$$
\mathrm{SNR}_{m}=\frac{N^{2}\left|d_{m}\right|^{2}}{\sigma^{2} \sum_{i=0}^{N-1}\left|S_{i}\right|^{-2}} .
$$

Notice that, we can obtain a larger $\mathrm{SNR}_{m}$ with a smaller value of $\sum_{i=0}^{N-1}\left|S_{i}\right|^{-2}$ by designing $S_{i}$. With the normalized energy constraint $\|\boldsymbol{S}\|^{2}=\sum_{i=0}^{N-1}\left|S_{i}\right|^{2}=1$, when $S_{i}$ has constant module for all $i$, i.e., $\left|S_{0}\right|=\left|S_{1}\right|=\cdots=\left|S_{N-1}\right|=\frac{1}{\sqrt{N}}$, we obtain the minimal value of $\sum_{i=0}^{N-1}\left|S_{i}\right|^{-2}=N^{2}$. In this case, the maximal SNR after the range compression can be obtained as

$$
\mathrm{SNR}_{\max , m}=\max _{\boldsymbol{S}:\|\boldsymbol{S}\|^{2}=1}\left\{\mathrm{SNR}_{m}\right\}=\frac{\left|d_{m}\right|^{2}}{\sigma^{2}} .
$$

Thus, the optimal signal $S_{i}$ should have constant module for all $i$, otherwise, the SNR after the range compression will be degraded. To evaluate the change of SNR, we define the SNR degradation factor as

$$
\xi=\frac{\mathrm{SNR}_{m}}{\mathrm{SNR}_{\max , m}}=\frac{N^{2}}{\sum_{i=0}^{N-1}\left|S_{i}\right|^{-2}} .
$$

Notice that although $\mathrm{SNR}_{m}$ and $\mathrm{SNR}_{\max , m}$ are both related to the $m$ th range cell in a swath, their ratio $\xi \in(0,1]$ is independent of the noise power $\sigma^{2}$ and $d_{m}$ and thus the above $\xi$ can be used to evaluate the SNR degradation after the range compression for all range cells. A larger $\xi$ denotes a less noise power enhancement (or a less SNR degradation) caused by the estimation processing in (13), and the generated signal $S_{i}$ is closer to the optimal one.

Since the length of the transmitted OFDM sequence $s_{t}$ is $N_{t}=N-M+1$, from the normalized energy constraint of $\boldsymbol{s}_{t}$, the mean transmitted power of $s_{t}$ is $\frac{1}{N_{t}}$. Thus, the SNR of the signal received from the $m$ th range cell before range compression is

$$
\overline{\mathrm{SNR}}_{m}=\frac{\left|d_{m}\right|^{2}}{N_{t} \sigma^{2}} .
$$

We notice that the maximal SNR of the $m$ th range cell after the range compression $\mathrm{SNR}_{\max , m}$ in (18) is equal to $N_{t} \overline{\mathrm{SNR}}_{m}$, and the range compression SNR gain is the same as that using LFM pulses with the same transmitted signal parameters [24], [25]. However, because of the sidelobes of the autocorrelation function using LFM pulses, the IRCI will occur in the range compression that degrades the signal-to-interference-plus-noise ratio (SINR). Considering the $M$ range cells in a swath, the interference of the $m$ th range cell from other range cells in the swath is

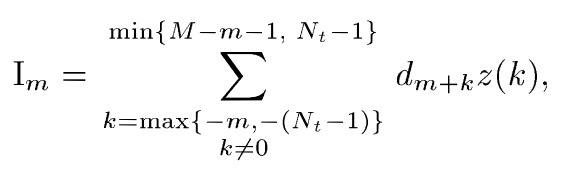

where $z(k)$ is the autocorrelation function of the LFM pulse, i.e.,

$$
z(k)=\sum_{n=0}^{N_{t}-1} l(n) l^{*}(n-k), \quad|k| \leq N_{t}-1,
$$

and $(\cdot)^{*}$ denotes the complex conjugate, $l(n), n=0, \ldots, N_{t}-$ 1 , are the values of a transmitted LFM sequence. $N_{t}$ denotes the length of the LFM sequence that is equal to the length of the OFDM sequence we use in this paper.

Thus, the SINR of the signal after the range compression using an LFM pulse is

$$
\operatorname{SINR}_{m}=\frac{\left|d_{m}\right|^{2}}{\left|\mathrm{I}_{m}\right|^{2}+\sigma^{2}} .
$$

To investigate the mean SINR, for convenience, we consider the mean power of range cells as $E\left[d_{m} d_{m}^{*}\right]=\sigma_{d}^{2}$. Then, the mean interference power, caused by the sidelobes, of each range cell in the swath is

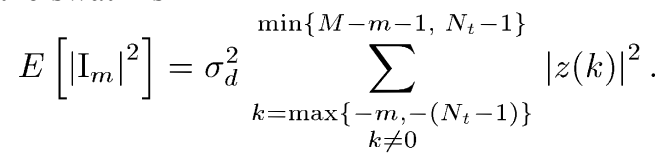

In this case, the mean SINR of the signal after the range compression using an LFM pulse is

$$
\mathrm{SINR}_{\mathrm{LFM}}=\frac{\sigma_{d}^{2}}{E\left[\left|\mathrm{I}_{m}\right|^{2}\right]+\sigma^{2}} .
$$

For given $M$ and $N_{t}$, SINR $_{\mathrm{LFM}}$ versus $\frac{\sigma_{d}^{2}}{\sigma^{2}}$ can be calculated using (24)-(25) and will be shown in the next section of simulations. Notice that since a random sequence has the same level of the sidelobe magnitudes of the autocorrelation values as an LFM signal does [21], the above SINR analysis also applies to the range compression in the random noise SAR imaging.

In contrast, for the IRCI free range compression by using an OFDM pulse, the SINR is equal to the SNR of the signal after the range compression, since for every range cell, there is no interrange-interference from other range cells. If the lower bound of the module of the OFDM sequence $S$ is $S_{\min }$, i.e., $\left|S_{i}\right|>S_{\min }$ for all $i=0,1, \ldots, N-1$, we can obtain

$$
\sum_{i=0}^{N-1}\left|S_{i}\right|^{-2}<N S_{\text {min }}^{-2} \text {. }
$$

Then, from (17), the SNR for the $m$ th range cell signal is lower bounded by

$$
\mathrm{SNR}_{m}=\frac{N^{2}\left|d_{m}\right|^{2}}{\sigma^{2} \sum_{i=0}^{N-1}\left|S_{i}\right|^{-2}}>\frac{N\left|d_{m}\right|^{2}}{\sigma^{2} S_{\mathrm{min}}^{-2}} .
$$

Thus, the SINR for all range cells after the range compression is also lower bounded by

$$
\mathrm{SINR}_{\mathrm{OFDM}}=E\left[\mathrm{SNR}_{m}\right]=\frac{N^{2} \sigma_{d}^{2}}{\sigma^{2} \sum_{i=0}^{N-1}\left|S_{i}\right|^{-2}}>\frac{N \sigma_{d}^{2}}{\sigma^{2} S_{\text {min }}^{-2}} .
$$

A remark to the lower bound for the SINR in (27) is that it does not depend on the swath width $M$, which is because our pro- 
posed OFDM SAR imaging algorithm with our proposed arbitrary length OFDM pulses is IRCI free and the pulse length does not depend on a swath width. Therefore, it is particularly interesting in wide swath SAR imaging applications. Based on the above analysis, the task here is to generate an OFDM sequence with a larger $\xi$ (or a less SNR degradation) by designing a sequence $S_{i}$ with larger $S_{\min }$. This motivates the following OFDM sequence design.

\section{New OFDM Sequence Design}

First of all, an OFDM pulse of any segment in (1) is determined by a weight sequence $\boldsymbol{S}=\left[S_{0}, S_{1}, \ldots, S_{N-1}\right]^{T}$ that is determined by its $N$-point IFFT $\boldsymbol{s}=\left[s_{0}, s_{1}, \ldots, s_{N-1}\right]^{T}$. Thus, an OFDM pulse design is equivalent to the design of its weight sequence $\boldsymbol{S}$ or the $N$-point IFFT, $\boldsymbol{s}$, of $\boldsymbol{S}$. From the studies in the preceding sections, an arbitrary length OFDM pulse $s(t)$ supported only in $\left[T_{G I}, T\right]$ for $T>T_{G I}$ with its sampled sequence $s_{n}=s\left(n T_{s}\right)$ should be designed as follows.

1) Sequence $s$ should satisfy the zero head condition in (5). When this condition is satisfied and the $N$-point FFT, $\boldsymbol{S}$, of $s$, is used as the weight sequence in the OFDM pulse in (1) denoted as $s_{1}(t)$, let its segment (or truncated version) only supported on $\left[T_{G I}, T\right]$ be denoted by $s(t)$ that is 0 for $t \in\left[0, T_{G I}\right) \cup\left(T, T+T_{G I}\right]$ and equals $s_{1}(t)$ for $t \in$ $\left[T_{G I}, T\right]$. Then, pulse $s(t)$ is still an OFDM pulse on its support and has length $T-T_{G I}$ of support (i.e., non-zero values) and this length can be arbitrary and independent of a swath width. Furthermore, $s(t)$ has the same discretetime sequence $\boldsymbol{s}^{\prime}$ as the OFDM pulse $s_{1}(t)$ does, which, thus, satisfies the zero head and tail condition (5). From the study in the preceding section, transmitting pulse $s(t)$ leads to the IRCI free range reconstruction in SAR imaging.

2) To avoid enhancing the noise as the estimation processing in (13) and achieve the maximal possible SNR after the range compression, the complex weights $S_{i}$ should be as constant module as possible for all $i$. In other words, $S_{\min }$ should be as large as possible.

3) The PAPR of the transmitted OFDM pulse $s(t)$ in (1) for $t \in\left[T_{G I}, T\right]$ should be minimized so that its transmitting and receiving can be implemented easier. Otherwise, a delta pulse would serve 1) and 2) above, but it has infinite bandwidth and infinite PAPR and can not be transmitted [25].

Unfortunately, it looks like that there is no closed-form solution of an OFDM sequence $s$ that simultaneously satisfies the above requirements 1)-3). It would be easy to have a sequence $\boldsymbol{s}=\left[s_{0}, s_{1}, \ldots, s_{N-1}\right]^{T}$ to satisfy the zero head condition in (5), i.e., $s_{n}=0$ for $n=0,1, \ldots, M-2$ as mentioned in the above 1). However, its FFT, $\boldsymbol{S}$, may not have constant module or may not be even close to constant module. A natural idea is to modify this sequence $S$ to be closer to constant module and then take its IFFT to go back to the time domain $s$ and also obtain the continuous waveform $s(t)$. Then, this $s$ may not satisfy the zero head condition in (5) anymore. Furthermore, the PAPR of the continuous waveform $s(t)$ for $t \in\left[T_{G I}, T\right]$ may be high. In this case, we may modify $s$ and in the meantime add some constraint to limit the PAPR of $s(t)$ for $t \in\left[T_{G I}, T\right]$. Our OFDM sequence design idea is to do the above process iteratively until

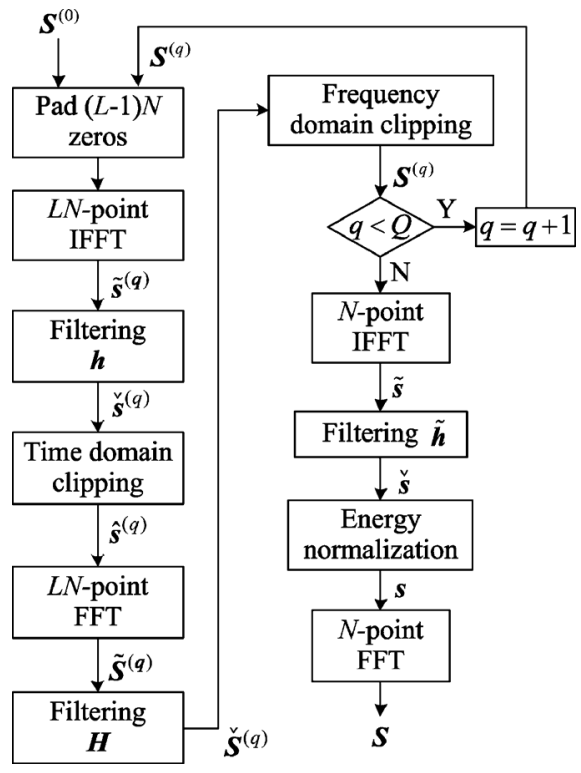

Fig. 2. Block diagram of the OFDM sequence design algorithm.

a pre-set iteration number is reached and/or a desired sequence $s$ is obtained.

To clearly describe the design algorithm, let us better understand the PAPR calculation for an analog waveform. For a sufficiently accurate PAPR estimation of a transmitted OFDM pulse, we usually consider its oversampled discrete time sequence, i.e., a time domain OFDM sequence $\tilde{\boldsymbol{s}}=\left[\tilde{s}_{0}, \ldots, \tilde{s}_{L N-1}\right]^{T}$ by $L$ times over-sampling of the continuous waveform $s(t)$ with complex weights $\boldsymbol{S}=\left[S_{0}, S_{1}, \ldots, S_{N-1}\right]^{T}$ in (1) for a sufficiently large $L$ [22], i.e.,

$$
\tilde{s}_{n}=\frac{1}{\sqrt{L N}} \sum_{i=0}^{N-1} S_{i} \exp \left\{\frac{j 2 \pi n i}{L N}\right\}, n=0, \ldots, L N-1,
$$

which can be implemented by the $L N$-point IFFT of the sequence $\left[S_{0}, S_{1}, \ldots, S_{N-1}, 0,0, \ldots, 0\right]^{T}$ of length $L N$.

Then, the PAPR of the transmitted OFDM pulse can be defined as

$$
\operatorname{PAPR}=\frac{\max _{n=0, \ldots, L N-1}\left|\tilde{s}_{n}\right|^{2}}{\frac{1}{L N} \sum_{n=0}^{L N-1}\left|\tilde{s}_{n}\right|^{2}} .
$$

Since $s$ and $S$ are FFT pairs, starting with $s$ and starting with $S$ are equivalent. For the convenience to deal with the PAPR issue, our proposed iterative algorithm starts with an initial random constant modular sequence $S^{(0)} \in \mathbb{C}^{N \times 1}$ and obtains $\tilde{\boldsymbol{s}}^{(q)} \in \mathbb{C}^{L N \times 1}$ using (28) as shown in Fig. 2.

Since the first $M-1$ samples of our desired sequence $s$ should be equal to zero, after the $L$ times over-sampling of the analog waveform, the first $L(M-1)$ samples in sequence $\tilde{s}_{n}^{(q)}, 0 \leq$ $n \leq L(M-1)-1$, should be equal to zero. Thus, we apply the following time domain filter to the newly obtained sequence $\tilde{\boldsymbol{s}}^{(q)}$ :

$$
h(n)=\left\{\begin{array}{ll}
0, & 0 \leq n \leq L(M-1)-1 \\
1, & L(M-1) \leq n \leq L N-1
\end{array},\right.
$$


as $\check{s}_{n}^{(q)}=\tilde{s}_{n}^{(q)} h(n), n=0, \ldots, L N-1$, to obtain a new sequence $\check{\boldsymbol{s}}^{(q)}=\left[\check{s}_{0}^{(q)}, \ldots, \check{s}_{L N-1}^{(q)}\right]^{T}$. After this truncation, we then add a PAPR constraint to the segment of the non-zero elements of this sequence to obtain the next new sequence $\hat{s}_{n}^{(q)}$ by clipping $\check{s}_{n}^{(q)}$ as follows. The time domain clipping can be defined as, [26],

$$
\begin{aligned}
& \hat{s}_{n}^{(q)}=\left\{\begin{array}{ll}
\mathrm{T}_{q} \frac{\check{s}_{n}^{(q)}}{\left|\check{s}_{n}^{(q)}\right|}, & \text { if }\left|\check{s}_{n}^{(q)}\right|>\mathrm{T}_{q} \\
\check{s}_{n}^{(q)}, & \text { if }\left|\check{s}_{n}^{(q)}\right| \leq \mathrm{T}_{q}
\end{array},\right. \\
& \mathrm{T}_{q}=\sqrt{\mathrm{PAPR}_{d} P_{t a v}^{(q)}},
\end{aligned}
$$

where $L(M-1) \leq n \leq L N-1$, and $\hat{s}_{n}^{(q)}=0$ for $n=$ $0, \ldots, L(M-1)-1$.

$$
P_{\text {tav }}^{(q)}=\frac{1}{L(N-M+1)} \sum_{n=L(M-1)}^{L N-1}\left|\check{s}_{n}^{(q)}\right|^{2}
$$

is the average power of the non-zero elements in sequence $\check{\boldsymbol{s}}^{(q)}$. $\mathrm{T}_{q}$ is the clipping level in the $q$ th iteration which is updated in each iteration according to the average power $P_{\text {tav }}^{(q)}$ and a constant value $\mathrm{PAPR}_{d}$ that is a lower bound for a desired PAPR.

After the $L N$-point FFT operation to $\hat{s}_{n}^{(q)}$, we obtain the frequency domain sequence $\tilde{\boldsymbol{S}}^{(q)}$. To constrain the out-of-band radiation caused by the time domain filtering and clipping, we also use a filter in the frequency domain:

$$
H(i)=\left\{\begin{array}{ll}
1, & 0 \leq i \leq N-1 \\
0, & N \leq i \leq L N-1
\end{array} .\right.
$$

And the output sequence $\check{S}_{i}^{(q)}$ can be obtained by $\check{S}_{i}^{(q)}=$ $\tilde{S}_{i}^{(q)} H(i), i=0, \ldots, L N-1$. To deal with the constant module issue of the frequency domain sequence $S$, then, the following frequency domain clipping is used:

$$
\begin{aligned}
& S_{i}^{(q+1)} \\
& = \begin{cases}\sqrt{P_{f a v}^{(q)}}\left(1+G_{f}\right) \frac{\check{S}_{i}^{(q)}}{\mid \check{S}_{i}^{(q)}}, & \text { if }\left|\check{S}_{i}^{(q)}\right|>\sqrt{P_{f a v}^{(q)}}\left(1+G_{f}\right) \\
\sqrt{P_{f a v}^{(q)}}\left(1-G_{f}\right) \frac{\check{S}_{i}^{(q)}}{\mid \check{S}_{i}^{(q)}}, & \text { if }\left|\check{S}_{i}^{(q)}\right|<\sqrt{P_{f a v}^{(q)}}\left(1-G_{f}\right), \\
\check{S}_{i}^{(q)}, & \text { otherwise }\end{cases}
\end{aligned}
$$

where $0 \leq i \leq N-1$, and sequence $S^{(q+1)}=$ $\left[S_{0}^{(q+1)}, S_{1}^{(q+1)}, \ldots, S_{N-1}^{(q+1)}\right]^{T}$ is obtained. And

$$
P_{f a v}^{(q)}=\frac{1}{N} \sum_{i=0}^{N-1}\left|\check{S}_{i}^{(q)}\right|^{2}
$$

is the average power of the non-zero elements in sequence $\check{\boldsymbol{S}}^{(q)}$. $G_{f}$ is a factor that we use to control the upper and lower bounds for sequence $S_{i}^{(q+1)}$. Thus, the module of sequence $S_{i}^{(q+1)}$ is constrained as $\left|S_{i}^{(q+1)}\right| \in \epsilon$ $\left[\sqrt{P_{f a v}^{(q)}}\left(1-G_{f}\right), \sqrt{P_{f a v}^{(q)}}\left(1+G_{f}\right)\right]$. A smaller $G_{f}$ denotes that a closer-to-constant modular sequence $\boldsymbol{S}^{(q+1)}$ can be obtained.
The above procedure is done for $q=0,1, \ldots$, when $q<Q$, where $Q$ is a pre-set maximum iteration number. When $q=Q$, the iteration stops and then $N$-point IFFT is applied to $\boldsymbol{S}^{(Q)} \in$ $\mathbb{C}^{N \times 1}$ to obtain $\tilde{\boldsymbol{s}} \in \mathbb{C}^{N \times 1}$. After that, a time domain filter, i.e.,

$$
\tilde{h}(n)=\left\{\begin{array}{ll}
0, & 0 \leq n \leq M-2 \\
1, & M-1 \leq n \leq N-1
\end{array},\right.
$$

is applied to $\tilde{\boldsymbol{s}}$ to obtain sequence $\check{\boldsymbol{s}}=\left[\check{s}_{0}, \ldots, \check{s}_{N-1}\right]^{T}$, where $\check{s}_{n}=\tilde{s}_{n} \tilde{h}(n), n=0, \ldots, N-1$. In order to normalize the energy of the sequence $s$ to 1, we use the normalization to the time domain sequence $\check{\boldsymbol{s}}$ as

$$
s_{n}=\frac{\check{s}_{n}}{\sqrt{\sum_{k=M-1}^{N-1}\left|\check{s}_{k}\right|^{2}}}, \quad n=0, \ldots, N-1,
$$

and obtain the OFDM sequence $\boldsymbol{s}$ in (6) that satisfies the zero head condition in (5). Finally, $\boldsymbol{S}$ can be obtained by taking the $N$-point FFT of $\boldsymbol{s}$. The PAPR of the non-zero part of $s_{n}$ for $M-1 \leq n \leq N-1$ can be calculated using (28) and (29) and the SNR degradation factor $\xi$ in (19) can also be calculated from $S$.

Notice that, after the last iteration, the filtering operation in time domain is applied to $\tilde{\boldsymbol{s}}$ to obtain $\boldsymbol{s}$, which will cause some out-of-band radiation to $S$. However, comparing to the OFDM sequence energy, the out-of-band radiation energy is much smaller and can be ignored as we shall see later in the simulations in the next section.

Therefore, for a given swath width and radar range resolution, we can obtain $M$. Then, for any $N$ with $N \geq M$, by using the above OFDM pulse design method, we can obtain an OFDM sequence $s$ with $M-1$ zeros at the head part of $s$ and $N-$ $M+1$ non-zero values in the remaining part of $\boldsymbol{s}$, and also its $N$-point FFT $S$. With this $S$ as the weights in (1), the OFDM pulse $s(t)$ in (1) for $t \in\left[T_{G I}, T\right]$ can be obtained. Since $N$ or correspondingly $T$ can be chosen arbitrarily, the pulse length, $T-T_{G I}$, of $s(t)$ can be arbitrary and independent of $M$ (or the swath width).

Let us go back to the mean SINR in (27) using OFDM pulses. Note that the constant module sequence $S_{i}$ is achieved when $\left|S_{i}\right|=\frac{1}{\sqrt{N}}$ for all $i, i=0,1, \ldots, N-1$. According to our numerous simulations, we find that it is not difficult to generate an OFDM sequence $S_{i}$ with $\left|S_{i}\right| \geq 0.8 \frac{1}{\sqrt{N}}, i=0, \ldots, N-1$, using our proposed OFDM pulse design algorithm above, which can be seen in the next section. Simulations about the above SINR comparison are also provided in the next section.

\section{Simulation Results}

In this section, by using simulations we first see the performance of our proposed OFDM sequence/pulse design of arbitrary length. We then see the performance of the IRCI free range reconstruction in SAR imaging with our proposed arbitrary length OFDM pulse.

\section{A. Performance of the OFDM Pulse Design}

In this subsection, we first discuss the performance of the OFDM pulse design algorithm. For simplicity, we set $M=96$ 


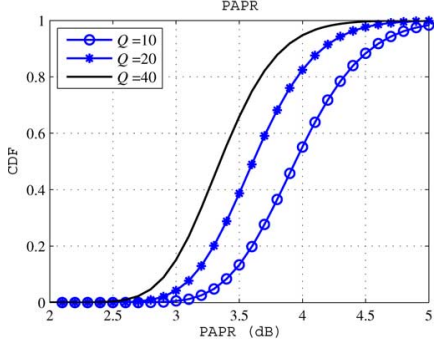

(a)

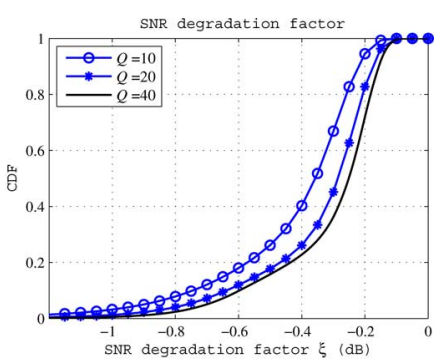

(b)
Fig. 3. The CDFs for different $Q$ with $\mathrm{PAPR}_{d}=1 \mathrm{~dB}$ and $G_{f}=5 \%$ : (a) PAPR; (b) SNR degradation factor.

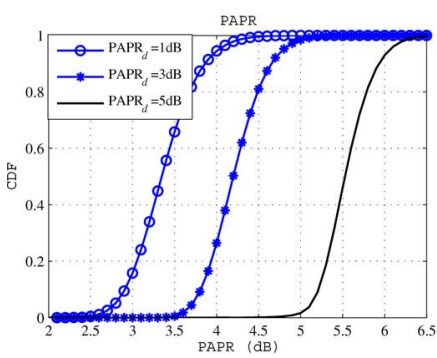

(a)

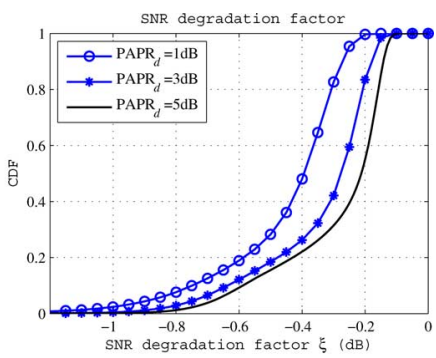

(b)
Fig. 4. The CDFs for different $\mathrm{PAPR}_{d}$ with $Q=20$ and $G_{f}=10 \%$ : (a) PAPR; (b) SNR degradation factor.

and $N=128$. To achieve a sufficiently accurate PAPR estimate, we set the over-sampling ratio $L=4$ [22]. Then, we can generate an OFDM sequence $s$ with $M-1=95$ zeros at the head part of $s$. We evaluate the PAPR and the SNR degradation factor $\xi$ by using the standard Monte Carlo technique with $5 \times 10^{5}$ independent trials. In each trial, the $i$ th element of initial sequence $\boldsymbol{S}^{(0)}$ is set as $S_{i}^{(0)}=e^{j 2 \pi \varphi_{i}}, i=0, \ldots, N-1$, where $\varphi_{i}$ is uniformly distributed over the interval $[0,2 \pi]$. In Figs. 3-5, we plot the cumulative distribution functions (CDF) of the PAPR and the SNR degradation factor $\xi$. The curves in Fig. 3 denote that, with the increase of the maximum iteration number $Q$, the PAPR decreases and the $\xi$ increases. In Fig. 3(a), more than $10 \%, 40 \%$ and $60 \%$ of the PAPRs of the OFDM sequences are less than $3.5 \mathrm{~dB}$ when $Q$ is equal to 10,20 and 40, respectively. In Fig. 3(b), the probability of $\xi>-0.4 \mathrm{~dB} \approx 0.91$, i.e., $\operatorname{Pr}(\xi>0.91)=1-\operatorname{Pr}(\xi \leq 0.91)$, is about $60 \%, 75 \%$ and $78 \%$ for $Q$ is equal to 10,20 and 40 , respectively. $\xi>-0.4 \mathrm{~dB} \approx 0.91$ denotes that the SNR of the received signal after the range reconstruction (using the designed OFDM pulse) is more than $91 \%$ of the maximum SNR using constant modular weights $S_{i}$. Thus, the SNR degradation of the CP-based SAR imaging algorithm can be insignificant by using our designed arbitrary length OFDM pulses. We also plot the CDFs for different $\mathrm{PAPR}_{d}$ with $Q=20$ and $G_{f}=10 \%$ in Fig. 4. The curves in Fig. 4 show that the PAPR change is more sensitive than the $\xi$ change for different $\mathrm{PAPR}_{d}$. Specifically, the curves in Fig. 4(a) indicate that the PAPR of a designed $s$ is significantly increased for the increase of $\mathrm{PAPR}_{d}$. And the curves in Fig. 4(b) denote that the SNR degradation becomes

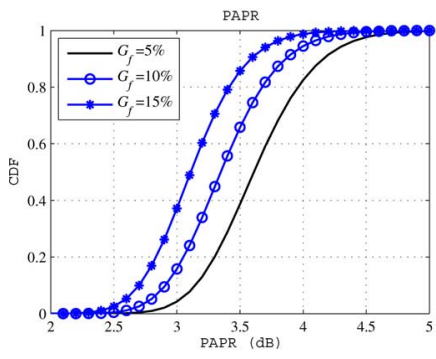

(a)

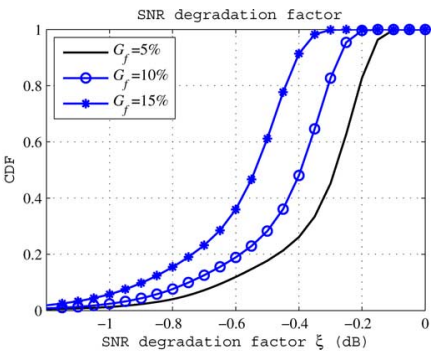

(b)
Fig. 5. The CDFs for different $G_{f}$ with $Q=20$ and $\mathrm{PAPR}_{d}=1 \mathrm{~dB}$ : (a) PAPR; (b) SNR degradation factor.

TABLE I

Numbers of MONTE CARLO TRIALS FOR $\xi$ AND PAPR WITH PAPR P $_{d}=1 \mathrm{~dB}$ AND $G_{f}=5 \%$

\begin{tabular}{|c|c|c|c|}
\hline & $\xi \geq-0.1 \mathrm{~dB}$ & $\xi \geq-0.2 \mathrm{~dB}$ & $\xi \geq-0.4 \mathrm{~dB}$ \\
\hline PAPR $\leq 2 \mathrm{~dB}$ & 4 & 5 & 7 \\
\hline PAPR $\leq 2.5 \mathrm{~dB}$ & 145 & 1511 & 2134 \\
\hline PAPR $\leq 3 \mathrm{~dB}$ & 615 & 35036 & 69735 \\
\hline \multicolumn{3}{|c|}{ Total number of trials: $5 \times 10^{5}$} \\
\hline
\end{tabular}

TABLE II

Numbers OF MONTE CARLO TRIALS For $S_{\min }$ With PAPR $_{d}=1 \mathrm{~dB}$ AND $G_{f}=5 \%$

\begin{tabular}{|c|c|c|c|}
\hline$S_{\min } \geq 0.88 \frac{1}{\sqrt{N}}$ & $S_{\min } \geq 0.85 \frac{1}{\sqrt{N}}$ & $S_{\min } \geq 0.8 \frac{1}{\sqrt{N}}$ & $S_{\min } \geq 0.5 \frac{1}{\sqrt{N}}$ \\
\hline 7 & 371 & 14415 & 353782 \\
\hline \multicolumn{3}{|c|}{ Total number of trials: $5 \times 10^{5}$} \\
\hline
\end{tabular}

less when $\mathrm{PAPR}_{d}$ is higher. Similarly, the curves in Fig. 5 indicate that the PAPR of $s$ is decreased and the SNR degradation is increased, when $G_{f}$ is increased.

In practice, we want to generate an OFDM sequence $s$ with the minimal PAPR as well as the minimal SNR degradation. However, according to the above analysis the PAPR and $\xi$ are interacting each other. Therefore, it is necessary to consider the constraints of both PAPR and $\xi$ at the same time. In Table I, we count the numbers of trials under different conditions of the PAPR and $\xi$ within the $5 \times 10^{5}$ Monte Carlo independent trials for $Q=40, \mathrm{PAPR}_{d}=1 \mathrm{~dB}$ and $G_{f}=5 \%$. Although only 4 trials meet the constraints of PAPR $\leq 2 \mathrm{~dB}$ and $\xi \geq-0.1 \mathrm{~dB}$, it can also indicate that an OFDM sequence with both low PAPR and low SNR degradation can be achieved by using our proposed OFDM pulse design algorithm. We also count the numbers of trials under different conditions of $S_{\min }$ in Table II. The number of trials for $S_{\min } \geq 0.8 \frac{1}{\sqrt{N}}$ are 14415, especially, there are 7 trials with $S_{\min } \geq 0.88 \frac{1}{\sqrt{N}}$. These results indicate that it is not difficult to generate an OFDM sequence $\boldsymbol{S}$ with $S_{\min } \geq 0.8 \frac{1}{\sqrt{N}}$. Specifically, a more excellent OFDM sequence with lower PAPR, larger $\xi$, and larger $S_{\min }$ can be obtained by doing more Monte Carlo trials or with a larger iteration number $Q$. Since in practice, the same OFDM pulse is used for SAR imaging and can be generated off-line, the computation time is not an issue here. As long as we find a desired design for a set of pre-designed radar parameters, it can be used forever for the radar system. In all of the above simulations, the out-of-band radiation energy of $\boldsymbol{S}$ is less than $10^{-30}$ and thus it can be completely ignored. 


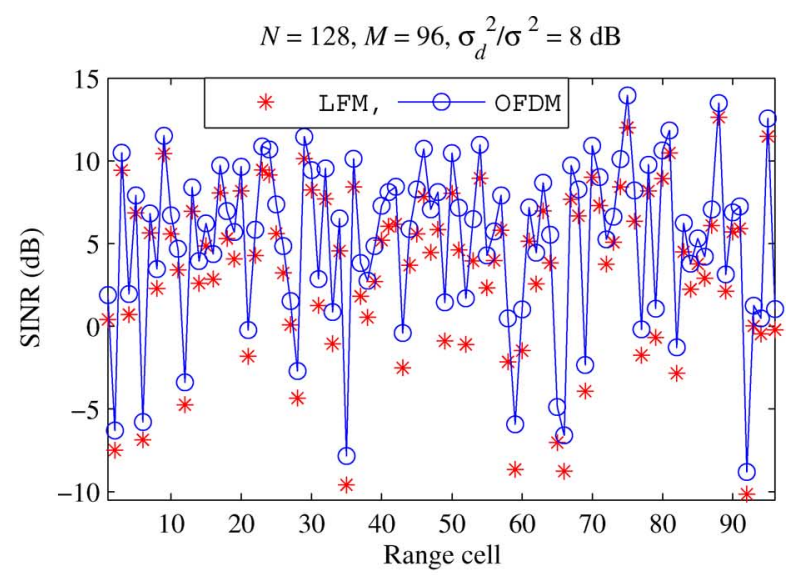

(a)

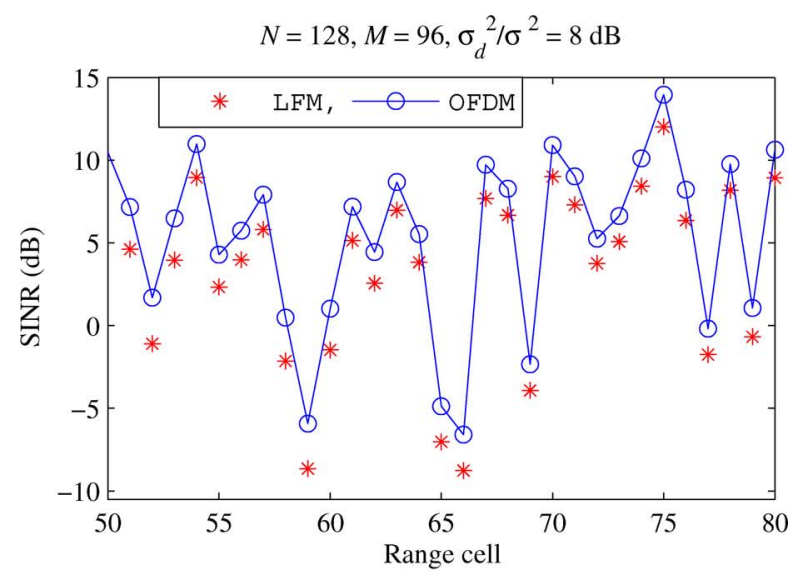

(b)

Fig. 6. SINRs after the range reconstructions using an LFM pulse and a designed OFDM pulse: (a) SINRs of all the $M$ range cells; (b) The zoom-in image of (a).

We also investigate the SINRs of the signals after the range reconstructions by using an LFM pulse and a designed OFDM pulse with $N=128$ in Fig. 6. The parameters of the LFM pulse are the same as the OFDM pulse, such as the transmitted pulse time duration, bandwidth and transmitted signal energy. We randomly choose a designed OFDM sequence with PAPR $=1.84 \mathrm{~dB}, \xi=-0.11 \mathrm{~dB}$ and $S_{\text {min }}=0.8 \frac{1}{\sqrt{N}}$. The randomly generated weighting RCS coefficients, $d_{m}, m=0, \ldots, M-1$, are included in $M=96$ range cells in a swath with $\frac{\sigma_{d}^{2}}{\sigma^{2}}=8 \mathrm{~dB}$. Then, the transmitted sequence length is $N_{t}=33$ that is independent of $M$. The SINRs of all the $M$ range cells are shown in Fig. 6(a). This figure indicates that the SINRs by using a designed OFDM pulse are larger than the SINRs by using an LFM pulse. The details from the 50th range cell to the 80th range cell are shown in its zoom-in image in Fig. 6(b).

In Fig. 7, we plot the SINRs after range reconstructions when using an LFM pulse as (25) as well as the SINRs and the lower bounds using the above designed OFDM pulse with $S_{\min }=$ $0.8 \frac{1}{\sqrt{N}}$ as $(27)$ versus $\frac{\sigma_{d}^{2}}{\sigma^{2}}$. The curves denote that the SINR lower bounds using the OFDM pulse are insignificantly smaller than the SINRs using the LFM pulse for $\frac{\sigma_{d}^{2}}{\sigma^{2}}<6 \mathrm{~dB}$. However, the SINR lower bounds using the OFDM pulse are larger than

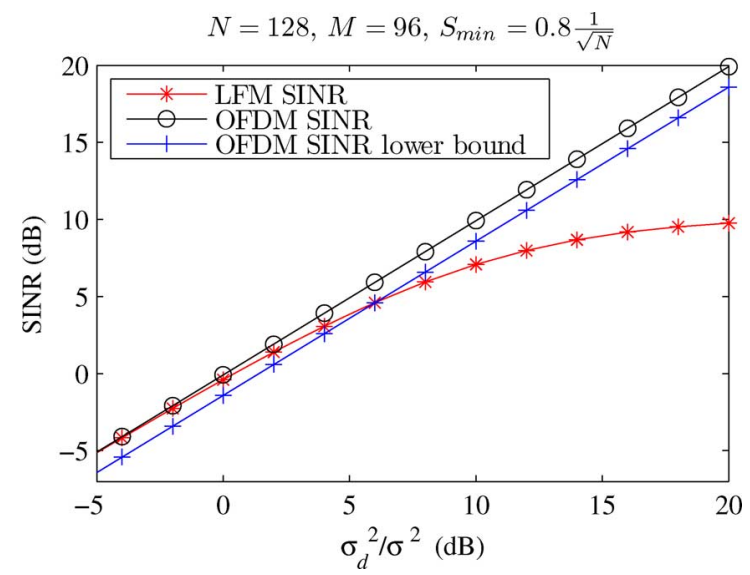

Fig. 7. Mean SINR comparison using an LFM pulse and a designed OFDM pulse.

the SINRs using the LFM pulse for $\frac{\sigma_{d}^{2}}{\sigma^{2}}>6 \mathrm{~dB}$. Moreover, the advantage of the SINR lower bounds by using the OFDM pulse is more obvious when $\frac{\sigma_{d}^{2}}{\sigma^{2}}$ is larger. Furthermore, the true SINRs using the OFDM pulse are about $1.4 \mathrm{~dB}$ larger than their lower bounds, never smaller than the SINRs using the LFM pulse for small $\frac{\sigma_{d}^{2}}{\sigma^{2}}$, and obviously larger than the SINRs using the LFM pulse for $\frac{\sigma_{d}^{2}}{\sigma^{2}}>0 \mathrm{~dB}$. These results indicate that the range reconstruction SNR degradation using a designed OFDM pulse is insignificant, and the advantage by using a designed OFDM pulse is more significant when noise power $\sigma^{2}$ becomes smaller.

\section{B. Performance of the SAR Imaging}

In this subsection, we present some simulations and discussions for the proposed CP-based arbitrary OFDM pulse length range reconstruction for SAR imaging. The azimuth processing is similar to the conventional stripmap SAR imaging [24], and a fixed value of $R_{c}$ located at the center of the range swath is set as the reference range cell for azimuth processing as what is commonly done in SAR image simulations. For comparison, we also consider the range Doppler algorithm (RDA) using LFM signals $^{2}$ as shown in the block diagram of Fig. 8. In Fig. 8(b), the secondary range compression (SRC) is implemented in the range and azimuth frequency domain, the same as the Option 2 in [24, Ch. 6.2].

The simulation parameters are set as in a typical SAR system: $\mathrm{PRF}=800 \mathrm{~Hz}$, the bandwidth is $B=150 \mathrm{MHz}$, the antenna length is $L_{a}=1 \mathrm{~m}$, the carrier frequency $f_{c}=9 \mathrm{GHz}$, the synthetic aperture time is $T_{a}=1 \mathrm{sec}$, the effective radar platform velocity is $v_{p}=150 \mathrm{~m} / \mathrm{sec}$, the platform height of the antenna is $H_{p}=5 \mathrm{~km}$, the slant range swath center is $R_{c}=5 \sqrt{2} \mathrm{~km}$, the sampling frequency $f_{s}=150 \mathrm{MHz}$.

Firstly, the normalized range profiles and azimuth profiles of a point spread function are shown in Fig. 9. It can be seen that the range sidelobes are much lower for the OFDM signal than

\footnotetext{
${ }^{2}$ Since the performance of random noise SAR is similar to LFM SAR, we do not present any simulation results of random noise SAR here. For more comparisons between OFDM SAR imaging, LFM SAR imaging, and random noise SAR imaging, we refer to [21].
} 


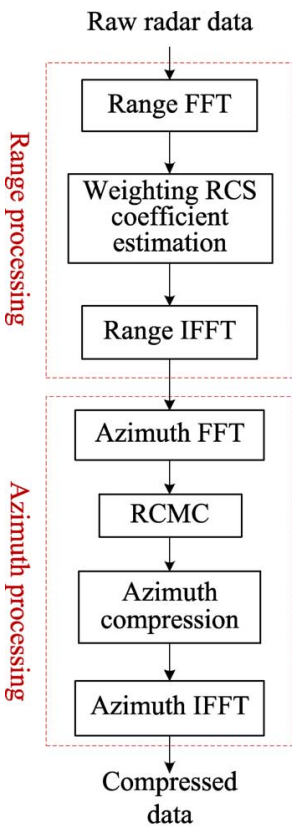

(a)

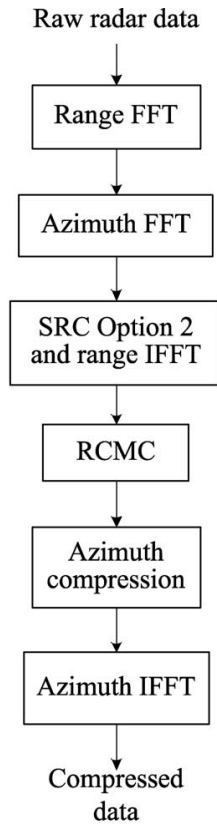

(b)

Fig. 8. Block diagram of SAR imaging processing: (a) CP-based OFDM SAR; (b) LFM SAR.

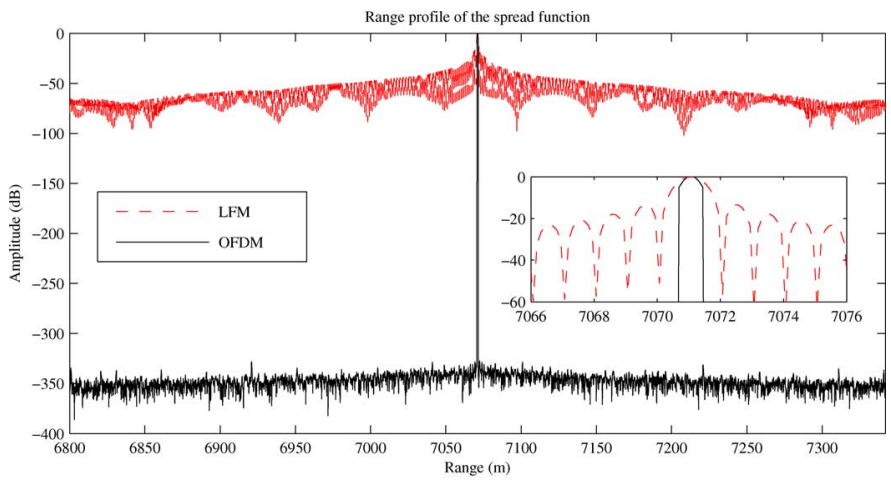

(a)

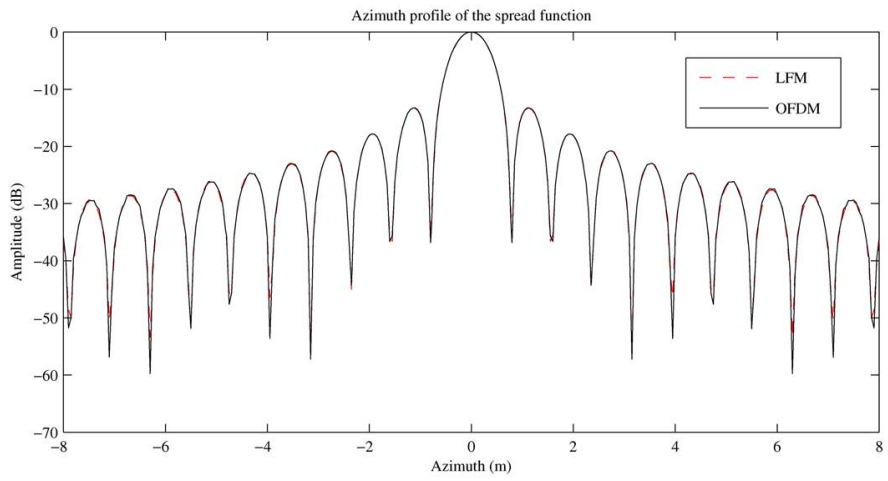

(b)

Fig. 9. Profiles of a point spread function: (a) range profiles; (b) azimuth profiles.

those of the LFM signal. And the azimuth profiles of the point spread function are similar for these two signals.

We also consider a single range line (a cross range) with $M=10000$ range cells in a $10 \mathrm{~km}$ wide swath, and targets

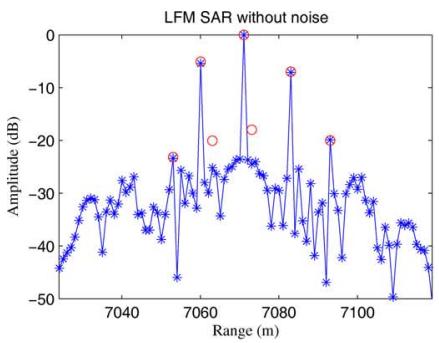

(a)

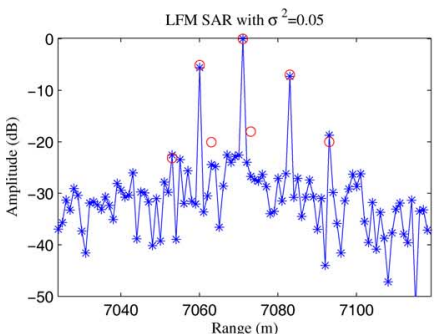

(c)

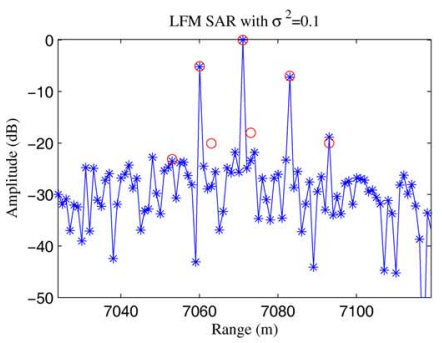

(e)

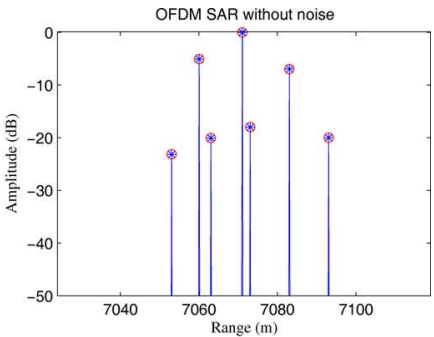

(b)

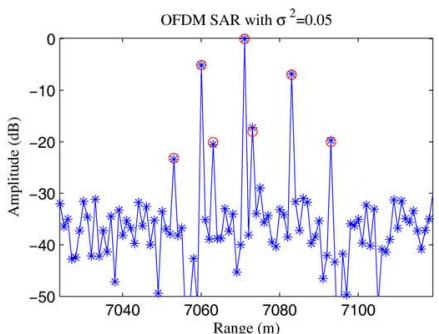

(d)

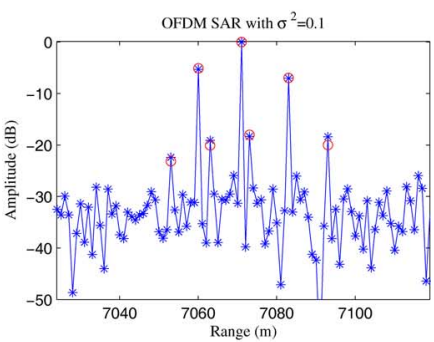

(f)
Fig. 10. A range line imaging results. Red circles denote the real target amplitudes, blue asterisks denote the imaging results. (a) LFM SAR without noise; (b) OFDM SAR without noise; (c) LFM SAR with noise of variance $\sigma^{2}=0.05$; (d) OFDM SAR with noise of variance $\sigma^{2}=0.05$; (e) LFM SAR with noise of variance $\sigma^{2}=0.1$; (f) OFDM SAR with noise of variance $\sigma^{2}=0.1$.

(non-zero RCS coefficients) are included in 7 range cells located from $7050 \mathrm{~m}$ to $7100 \mathrm{~m}$, the amplitudes are randomly generated and shown as the red circles in Fig. 10, and the RCS coefficients of the other range cells are set to be zero (for a better display, only a segment of the swath is indicated in Fig. 10). In this simulation, we use a designed OFDM pulse with PAPR $=1.93 \mathrm{~dB}, \xi=-0.14 \mathrm{~dB}$ and time dura$\operatorname{tion}^{3} T-T_{G I}=5 \mu \mathrm{s}$, which is independent of the swath width. For $T_{G I}=\frac{M-1}{f_{s}}, N=T f_{s}=10749$. The transmitted LFM pulse duration is also $5 \mu \mathrm{s}$. The normalized imaging results are shown as the blue asterisks in Fig. 10. The imaging results without noise are shown in Fig. 10(a) and (b). Since there is no IRCI between different range cells, the results indicate that the OFDM SAR imaging is precise as shown in Fig. 10(b). However, because of the influence of range sidelobes of the LFM signal, some weak targets, for example, those located at $7063 \mathrm{~m}$ and $7073 \mathrm{~m}$, are submerged by the interference from the nearby targets and thus can not be imaged correctly as shown in Fig. 10(a). We also give the imaging results of LFM SAR and OFDM SAR in Fig. 10(c) and (d), respectively, when the noise power of the raw radar data is $\sigma^{2}=0.05$,

${ }^{3}$ For the algorithm in [21], by setting $N=M$, the OFDM pulse time duration with sufficient length $C P$ is at least $T+T_{G I}=\frac{10000}{150}+\frac{9999}{150} \mu \mathrm{s} \approx 133.3 \mu \mathrm{s}$ as mentioned in Section II. 
and in Fig. 10(e) and (f), respectively, when $\sigma^{2}=0.1$. We highlight that the SNRs of the 7 targets of the raw radar data are $[-32.9,-15,-30.1,-10.2,-27.9,-17.1,-30] \mathrm{dB}$ for $\sigma^{2}=0.05$. These results can also indicate the better performance of the proposed OFDM SAR. The performance advantage of the OFDM SAR is more obvious for a smaller noise power, for example, when $\sigma^{2}=0.05$, which is consistent with the results in Fig. 7. Note that, for a better display and recognizability, we consider that only 7 range cells in the swath contain targets. In a practical SAR imaging, much more targets (non-zero RCS coefficients) are included and then the IRCI of LFM (or random noise) SAR will be more serious. Thus, the performance advantage of the OFDM SAR over LFM or random noise SAR will be more obvious because of its IRCI free range reconstruction.

\section{CONCLUSION}

In this paper, we proposed a novel sufficient CP-based OFDM SAR imaging algorithm with arbitrary pulse length that is independent of a swath width by using our newly proposed and designed OFDM pulses. This OFDM SAR imaging algorithm can provide the advantage of IRCI free range reconstruction and avoid the energy redundancy. We first established the arbitrary pulse length OFDM SAR imaging system model and then derived the range reconstruction algorithm with free IRCI. We also analyzed the SINR after the range reconstruction and compared it with that using LFM signals. By considering the PAPR of a transmitted OFDM pulse and the SNR degradation of the range reconstruction, we proposed a novel OFDM pulse design method. We finally gave some simulations to demonstrate the performance of the proposed OFDM pulse design method. By comparing with the RDA SAR imaging using LFM signals, we provided some simulations to illustrate the advantage, such as higher SINR after the range reconstruction, of the proposed arbitrary pulse length OFDM SAR imaging algorithm. The main contributions of this paper can be summarized as:

- When a sufficient CP length is at least $M-1$, where $M$ is the number of range cells within a swath, an OFDM sequence of length $N, s \in \mathbb{C}^{N \times 1}$, with at least $M-1$ consecutive zero elements in the head part is generated by an OFDM pulse design method and thus, the transmitted OFDM sequence is $\boldsymbol{s}_{t} \in \mathbb{C}^{(N-M+1) \times 1}$ of length $N-M+$ 1.

- With our proposed OFDM sequence/pulse design, a transmitted OFDM pulse length can be arbitrary and independent of a swath width, which is critical in wide swath IRCI free SAR imaging applications.

- With a designed OFDM pulse, no CP in the transmitted sequence needs to be removed in the receiver. Thus, the transmitted energy redundancy can be avoided.

- The proposed SAR imaging algorithm may cause some SNR degradation. However, the degradation is insignificant according to our simulations. Comparing with LFM SAR, the performance advantage of the OFDM SAR is more obvious for a smaller noise power. Moreover, with our proposed OFDM pulse design method, a better OFDM sequence with a lower PAPR can be generated by setting a larger maximum iteration number $Q$, and the SNR degradation by using this OFDM sequence becomes less.

\section{REFERENCES}

[1] N. Levanon, "Multifrequency complementary phase-coded radar signal," IEE Proc. Radar, Sonar Navig., vol. 147, no. 6, pp. 276-284, 2000.

[2] S. Sen and A. Nehorai, "Target detection in clutter using adaptive OFDM radar," IEEE Signal Process. Lett., vol. 16, no. 7, pp. 592-595, 2009.

[3] S. Sen and A. Nehorai, "Adaptive OFDM radar for target detection in multipath scenarios," IEEE Trans. Signal Process., vol. 59, no. 1, pp. 78-90, 2011.

[4] S. Sen, "PAPR-constrained Pareto-Optimal waveform design for OFDM-STAP radar," IEEE Trans. Geosci. Remote Sens., vol. 52, no. 6, pp. 3658-3669, 2014.

[5] S. Sen and A. Nehorai, "OFDM MIMO radar with mutual-information waveform design for low-grazing angle tracking," IEEE Trans. Signal Process., vol. 58, no. 6, pp. 3152-3162, 2010.

[6] D. Garmatyuk, J. Schuerger, K. Kauffman, and S. Spalding, "Wideband OFDM system for radar and communications," in Proc. IEEE Radar Conf., Pasadena, CA, 2009, pp. 1-6.

[7] C. Berger, B. Demissie, J. Heckenbach, P. Willett, and S. Zhou, "Signal processing for passive radar using OFDM waveforms," IEEE J. Sel. Topics Signal Process., vol. 4, no. 1, pp. 226-238, 2010.

[8] F. Colone, K. Woodbridge, H. Guo, D. Mason, and C. Baker, "Ambiguity function analysis of wireless LAN transmissions for passive radar," IEEE Trans. Aerosp. Electron. Syst. , vol. 47, no. 1, pp. 240-264, 2011.

[9] P. Falcone, F. Colone, C. Bongioanni, and P. Lombardo, "Experimental results for OFDM WiFi-based passive bistatic radar," in Proc. IEEE Radar Conf., Washington, DC, USA, 2009, pp. 516-521.

[10] F. Colone, P. Falcone, and P. Lombardo, "Ambiguity function analysis of WiMAX transmissions for passive radar," in Proc. IEEE Radar Conf., Washington, DC, USA, 2010, pp. 689-694.

[11] K. Chetty, K. Woodbridge, H. Guo, and G. Smith, "Passive bistatic WiMAX radar for marine surveillance," in Proc. IEEE Radar Conf., Washington, DC, USA, 2010, pp. 188-193.

[12] Q. Wang, C. Hou, and Y. Lu, "WiMAX signal waveform analysis for passive radar application," in Proc. RADAR. Int. Radar Conf.-Surv. Safer World, Bordeaux, France, 2009, pp. 1-6.

[13] V. Riche, S. Meric, J. Baudais, and E. Pottier, "Optimization of OFDM SAR signals for range ambiguity suppression," in Proc. 9th Eur. Radar Conf. (EuRAD), Amsterdam, The Netherlands, 2012, pp. 278-281.

[14] V. Riche, S. Meric, E. Pottier, and J.-Y. Baudais, "OFDM signal design for range ambiguity suppression in SAR configuration," in Proc. IEEE Int. Geosci. Remote Sens. Symp. (IGARSS), Munich, Germany, 2012, pp. $2156-2159$

[15] V. Riche, S. Meric, J.-Y. Baudais, and E. Pottier, "Investigations on OFDM signal for range ambiguity suppression in SAR configuration," IEEE Trans. Geosci. Remote Sens., vol. 52, no. 7, pp. 4194-4197, 2014.

[16] J.-H. Kim, M. Younis, A. Moreira, and W. Wiesbeck, "A novel OFDM chirp waveform scheme for use of multiple transmitters in SAR," IEEE Geosci. Remote Sens. Lett., vol. 10, no. 3, pp. 568-572, 2013.

[17] D. Garmatyuk, "Simulated imaging performance of UWB SAR based on OFDM," in Proc. IEEE Int. Conf. Ultra-Wideband, Waltham, MA, USA, 2006, pp. 237-242.

[18] D. Garmatyuk and M. Brenneman, "Adaptive multicarrier OFDM SAR signal processing," IEEE Trans. Geosci. Remote Sens., vol. 49, no. 10, pp. 3780-3790, 2011.

[19] D. Garmatyuk, "Cross-range SAR reconstruction with multicarrie OFDM signals," IEEE Geosci. Remote Sens. Lett., vol. 9, no. 5, pp. 808-812, 2012.

[20] J. R. Gutierrez Del Arroyo and J. A. Jackson, "WiMAX OFDM for passive SAR ground imaging," IEEE Trans. Aerosp. Electron. Syst., vol. 49, no. 2, pp. 945-959, 2013.

[21] T. Zhang and X.-G. Xia, "OFDM synthetic aperture radar imaging with sufficient cyclic prefix," IEEE Trans. Geosci. Remote Sens., vol. 53, no. 1, pp. 394-404, 2015.

[22] S. H. Han and J. H. Lee, "An overview of peak-to-average power ratio reduction techniques for multicarrier transmission," IEEE Wireless Commun., vol. 12, no. 2, pp. 56-65, 2005.

[23] R. Prasad, OFDM for Wireless Communications Systems. Boston: Artech House, 2004.

[24] M. Soumekh, Synthetic Aperture Radar Signal Processing. New York, NY, USA: Wiley, 1999 
[25] M. I. Skolnik, Introduction to Radar Systems. New York, NY, USA: McGraw-Hill, 2001.

[26] J. Armstrong, "Peak-to-average power reduction for OFDM by repeated clipping and frequency domain filtering," Electron. Lett., vol. 38 , no. 5 , pp. 246-247, 2002.

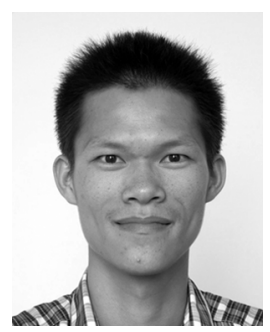

Tianxian Zhang received his B.S. degree from the University of Electronic Science and Technology of China (UESTC) in 2009. He is currently working toward his Ph.D. degree on signal and information processing at UESTC.

Since October 2012, he has been a visiting student researcher at the University of Delaware, Newark. His current research interests include radar and statistical signal processing, and synthetic aperture radar (SAR) systems.

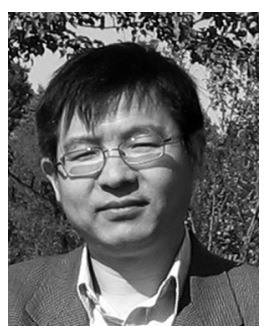

Xiang-Gen Xia (M'97-S'00-F'09) received his B.S. degree in mathematics from Nanjing Normal University, Nanjing, China, and his M.S. degree in mathematics from Nankai University, Tianjin, China, and his Ph.D. degree in electrical engineering from the University of Southern California, Los Angeles, in 1983, 1986, and 1992, respectively.

$\mathrm{He}$ was a Senior/Research Staff Member at Hughes Research Laboratories, Malibu, CA, during 1995-1996. In September 1996, he joined the Department of Electrical and Computer Engineering, University of Delaware, Newark, where he is the Charles Black Evans Professor. His current research interests include space-time coding, MIMO and

OFDM systems, digital signal processing, and synthetic aperture radar (SAR) and inverse synthetic aperture radar (ISAR) imaging. He has over 270 refereed journal articles published and accepted, is the holder of seven U.S. patents, and is the author of the book Modulated Coding for Intersymbol Interference Channels (New York: Marcel Dekker, 2000).

Dr. Xia received the National Science Foundation (NSF) Faculty Early Career Development (CAREER) Program Award in 1997, the Office of Naval Research (ONR) Young Investigator Award in 1998, and the Outstanding Overseas Young Investigator Award from the National Nature Science Foundation of China in 2001. He also received the Outstanding Junior Faculty Award of the Engineering School of the University of Delaware in 2001. He is currently serving and has served as an Associate Editor for numerous international journals including IEEE TRANSACtions on Signal Processing, IEEE TRANSACTIONS ON WIRELESS COMMUNICATIONS, IEEE TRANSACTIONS ON Mobile Computing, and IEEE Transactions on Vehicular TeChNOLOGy. $\mathrm{He}$ is Technical Program Chair of the Signal Processing Symp., Globecom 2007 in Washington DC, and the General Co-Chair of International Conference on Acoustics, Speech and Signal Processing (ICASSP) 2005 in Philadelphia.

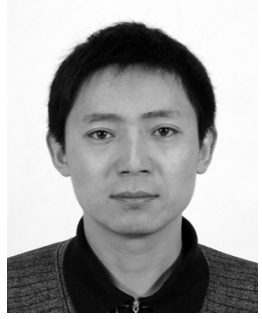

processing.
Lingjiang Kong was born in 1974. He received the B.S., M.S., and Ph.D. degrees from the University of Electronic Science and Technology of China (UESTC) in 1997, 2000 and 2003, respectively.

From September 2009 to March 2010, he was a visiting researcher with the University of Florida. He is currently a professor with the School of Electronic Engineering, University of Electronic Science and Technology of China (UESTC). His research interests include multiple-input multiple-output (MIMO) radar, through the wall radar, and statistical signal 